\title{
GSVMA: A Genetic Support Vector Machine ANOVA Method for CAD Diagnosis
}

\author{
Javad Hassannataj Joloudari ${ }^{1 *}$, Faezeh Azizi ${ }^{1}$, Mohammad Ali Nematollahi ${ }^{2}$, \\ Roohallah Alizadehsani ${ }^{3}$, Edris Hassannatajjeloudari ${ }^{4}$, Issa Nodehi ${ }^{5}$ and \\ Amir Mosavi ${ }^{6,7,8,9,10 *}$
}

\begin{abstract}
${ }^{1}$ Department of Computer Engineering, Faculty of Engineering, University of Birjand, Birjand, Iran, ${ }^{2}$ Department of Computer Sciences, Fasa University, Fasa, Iran, ${ }^{3}$ Institute for Intelligent Systems Research and Innovation, Deakin University, Geelong, VIC, Australia, ${ }^{4}$ Department of Nursing, School of Nursing and Allied Medical Sciences, Maragheh Faculty of Medical Sciences, Maragheh, Iran, ${ }^{5}$ Department of Computer Engineering, University of Qom, Qom, Iran, ${ }^{6}$ Faculty of Informatics, Technische Universität Dresden, Dresden, Germany, ${ }^{7}$ Faculty of Civil Engineering, TU-Dresden, Dresden, Germany, ${ }^{8}$ John von Neumann Faculty of Informatics, Óbuda University, Budapest, Hungary, ${ }^{9}$ Institute of Information Society, University of Public Service, Budapest, Hungary, ${ }^{10}$ Institute of Information Engineering, Automation and Mathematics, Slovak University of Technology in Bratislava, Bratislava, Slovakia
\end{abstract}

\section{OPEN ACCESS}

Edited by:

Stéphane Cook,

Université de Fribourg, Switzerland

Reviewed by:

Rajiv Rampat,

William Harvey Hospital,

United Kingdom

Dominique Monlezun,

University of Texas MD Anderson

Cancer Center, United States

*Correspondence:

Javad Hassannataj Joloudari

javad.hassannataj@birjand.ac.ir

Amir Mosavi

amir.mosavi@mailbox.tu-dresden.de

Specialty section:

This article was submitted to

Coronary Artery Disease,

a section of the journal

Frontiers in Cardiovascular Medicine

Received: 17 August 2021 Accepted: 22 December 2021

Published: 04 February 2022

Citation:

Hassannataj Joloudari J, Azizi F, Nematollahi MA, Alizadehsani $R$, Hassannatajjeloudari E, Nodehi I and Mosavi A (2022) GSVMA: A Genetic

Support Vector Machine ANOVA

Method for CAD Diagnosis.

Front. Cardiovasc. Med. 8:760178.

doi: 10.3389/fcvm.2021.760178
Background: Coronary artery disease $(C A D)$ is one of the crucial reasons for cardiovascular mortality in middle-aged people worldwide. The most typical tool is angiography for diagnosing CAD. The challenges of CAD diagnosis using angiography are costly and have side effects. One of the alternative solutions is the use of machine learning-based patterns for CAD diagnosis.

Methods: Hence, this paper provides a new hybrid machine learning model called genetic support vector machine and analysis of variance (GSVMA). The analysis of variance (ANOVA) is known as the kernel function for the SVM algorithm. The proposed model is performed based on the Z-Alizadeh Sani dataset so that a genetic optimization algorithm is used to select crucial features. In addition, SVM with ANOVA, linear SVM (LSVM), and library for support vector machine (LIBSVM) with radial basis function (RBF) methods were applied to classify the dataset.

Results: As a result, the GSVMA hybrid method performs better than other methods. This proposed method has the highest accuracy of $89.45 \%$ through a 10 -fold crossvalidation technique with 31 selected features on the Z-Alizadeh Sani dataset.

Conclusion: We demonstrated that SVM combined with genetic optimization algorithm could be lead to more accuracy. Therefore, our study confirms that the GSVMA method outperforms other methods so that it can facilitate CAD diagnosis.

Keywords: coronary artery disease, genetic algorithm, support vector machine, machine learning, diagnosis

\section{INTRODUCTION}

Cardiovascular disease (CVD) is one of the most prevalent diseases which cause a lot of deaths worldwide (1). As crucial evidence for this fact, one can refer to the CVD fact sheet published by the World Health Organization (WHO), which estimated 17.9 million deaths from CVDs in 2019, representing 32\% of all global deaths. Of these deaths, $85 \%$ were due to heart attack and stroke (2). An essential type of CVDs is coronary artery disease (CAD) (3). One of the reasons that made CAD such a necessary and stressful disease is the fact that nearly $25 \%$ of people who have been diagnosed with CAD died unexpectedly without any prior symptoms (4). 
Nowadays, electrocardiogram, cardiac stress test, coronary computed tomographic angiography, and coronary angiogram are some of the prevalent techniques used as diagnostic methods for CAD. The downside facts about all these methods are having side effects and imposing high costs on patients and health systems. Hence, today, applying machine learning methods for diagnosing $\mathrm{CAD}$ has become a general tendency. These techniques are important for modeling and knowledge extraction from row dataset (5).

To evaluate the performance of these new techniques, various $\mathrm{CAD}$ datasets have been prepared. Among these datasets, the Z-Alizadeh Sani dataset, Cleveland, and Hungarian are public.

In recent years, studies have been presented using machine learning methods for CAD diagnosis on different datasets. The well-known dataset, namely the Z-Alizadeh Sani dataset in the field of heart disease, is utilized. It is worth noting that until now, dozens of studies on the Z-Alizadeh Sani dataset have been published (5-25). The main goal of recent studies is to utilize feature selection methods to improve the accuracy of CAD diagnosis. In (10), a classification accuracy of 87.85 was obtained for CAD diagnosis by ANN classifier, so that 25 features were identified. In (14), a hybrid model titled nested ensemble nusupport vector classification method was presented to predict CAD. An accuracy of $94.66 \%$ was obtained using the hybrid method on the Z-Alizadeh Sani dataset so that 16 features were selected.

In a recent study (25), the CAD diagnosis was conducted using the weighted-average voting ensemble method. An accuracy of 98.97\% was achieved using the ensemble method on five features.

We obtained the highest area under the curve (AUC) and accuracy with more valuable and important features.

The previous studies demonstrate that Support Vector Machine (SVM) performs better for binary classification and dimension reduction on a small dataset $(16,26)$.

Hence, we utilized the SVM method with kernel types such as analysis of variance (ANOVA), linear SVM (LSVM), and library for support vector machine (LIBSVM) with radial basis function (RBF) on the Z-Alizadeh Sani dataset. Also, a genetic algorithm as an optimizer is used to select important features in the SVM modeling process. Ultimately, among the proposed methods used in this paper, the genetic optimizer method combined with SVM and ANOVA kernel has the most accuracy of $89.45 \%$ on 31 features.

In summary, the main contributions of our paper are as follows:

1) Performing data preprocessing (transforming nominal data to numerical data and normalization)

2) Using genetic algorithm as a feature selection method for selecting important features

3) Specifying ANOVA kernel as the best kernel compared to the other kernels

4) Generating the hybrid model consist of genetic training for feature selection, SVM for classification, and a 10-fold crossvalidation technique

5) Obtaining a maximum AUC of $100 \%$ on the Z-Alizadeh Sani dataset

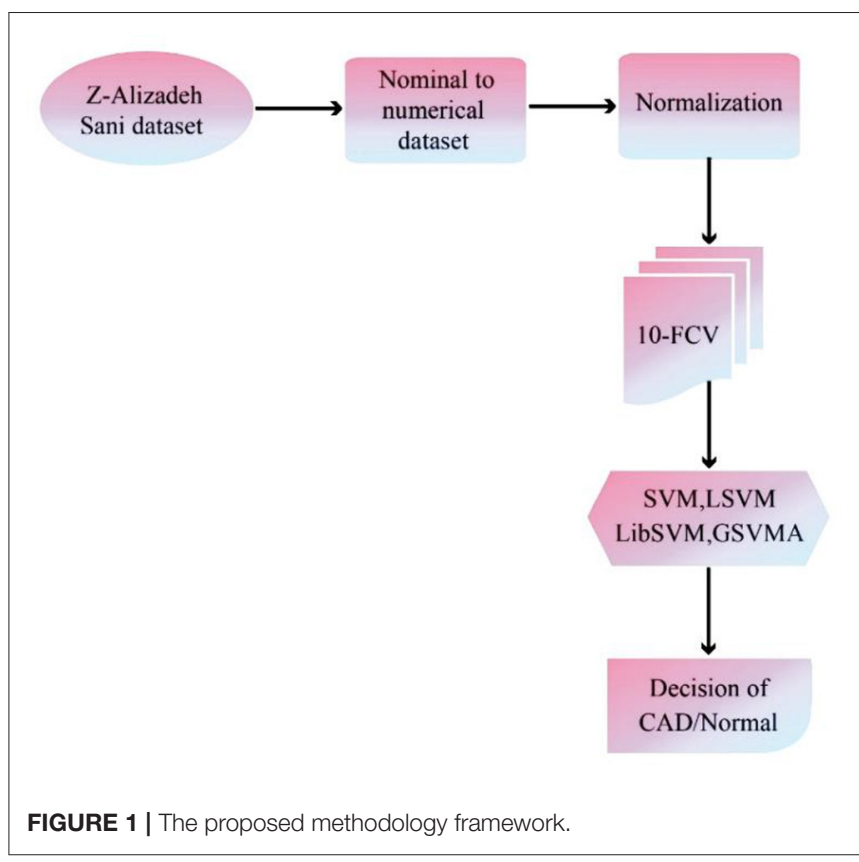

\section{MATERIALS AND METHODS}

The proposed methodology has been performed in three subsections. Section Z-Alizadeh Sani Dataset describes the ZAlizadeh Sani dataset. Also, in section Data Preprocessing, data preprocessing will be done. In addition, data classification using SVM with ANOVA, LSVM, and LIBSVM with RBF and GSVMA methods is described in section Used Classification Methods. The proposed methodology framework is shown in Figure 1.

\section{Z-Alizadeh Sani Dataset}

The Z-Alizadeh Sani dataset is one of the most common datasets used in machine learning for automatic CAD detection. This dataset is constructed from 303 patients referred to Shaheed Rajaie Cardiovascular, Medical, and Research Center ${ }^{1}$ A patient is categorized as a patient with CAD if one or more of his/her coronary arteries are stenosis. A coronary artery is categorized as stenosis if its diameter narrowing is $\geq 50 \%$ (27). Accordingly, 216 patients had $\mathrm{CAD}$, and the dataset contains 88 patients with the normal situation on the Z-Alizadeh Sani. Each record in this dataset has 55 features that can be used as indicators of CAD for a patient. These features are grouped into four categories include demographic, symptom and examination, laboratory and echo, and ECG features as explained in Table $\mathbf{1}$.

\section{Data Preprocessing}

In the data analysis process, preprocessing is required after data gathering. The Z-Alizade Sani dataset was numerical and string. First, the values of features are transformed from nominal data to numerical data. The features such as sex, chronic renal failure, cerebrovascular accident, airway disease, thyroid disease, congestive heart failure, dyslipidemia, etc., are

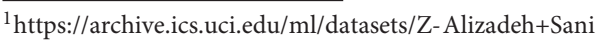


TABLE 1 | Description of the Z-Alizadeh-Sani dataset (5).

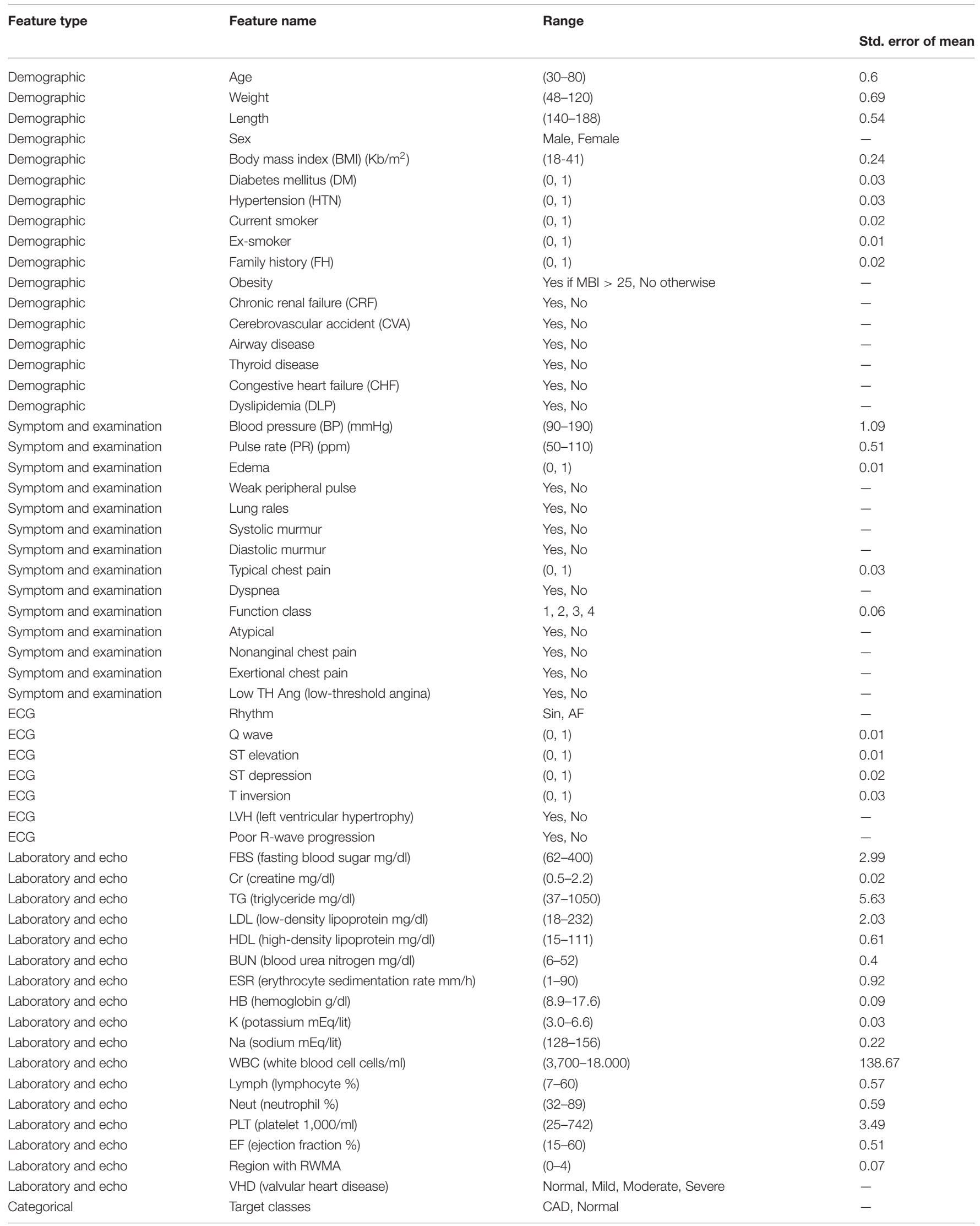

Std: Standard. 
transformed. Then, the data normalization is performed. The range transformation technique is a common technique for normalizing data related to features between 0 and 1 (5). In other words, changing the range of data to zero and one means changing the mean and variance to mean zero and variance 1. Normalizing the data helps all features have an equal effect and role in diagnosing the input class so that normalizing efforts to allow all features an equal weight.

The values of features such as diabetes mellitus (DM), hypertension (HTN), current smoker, ex-smoker, etc., are transformed between zero and one. In general, normalization leads to an increase in the accuracy of the classification methods. Furthermore, a 10-fold crossvalidation (10-FCV) technique (28, 29) for partitioning the dataset was utilized so that the dataset was divided into training (90\%) and test (10\%) subsets. The 10-FCV process was run 10 times in which the results of the methods were obtained by averaging every 10 times.

\section{Used Classification Methods \\ SVM}

For the first time, the SVM algorithm has been developed for data classification in (30-32), which is an optimal selection when robust predictive power is required. The SVM is a supervised machine learning algorithm that transforms data to a high dimensional space, that is, Hilbert space. Then kernel-based methods due to the visions presented by the generalization theory are exploited, and the optimization theory is performed (33). Indeed, SVM is an area parting model in which the data allocated into the support vectors are based on machine learning and model construction $(34,35)$.

In general, the SVM aims to find the best separator line (optimal hyperplane) between the data of the two classes so that it has the most significant possible distance from all the support vectors of the two classes. These classes are partitioned as linear and nonlinear statuses $(34,36)$. In these statuses, the SVM is considered that there is a set of training data $x_{1}, x 2, \ldots, x 3 € R^{n}$ with class $y_{1} €\{1,-1\}$ that are binary $\left(x_{i}, y_{i}\right),(i=1,2, \ldots n)$, and $n$ represents the number of training data points.

In this paper, we used methods such as LSVM, library SVM with RBF, SVM with ANOVA, and genetic support vector machine with ANOVA (GSVMA). RapidMiner software version 9.9 has been used to implement the methods. We described these methods in the following.

\section{Linear SVM}

The linear kernel is the most common kernel function for LSVM (37). The LSVM model generates an optimized hyperplane that discrete the data points of the two classes.

For an LSVM, a decision function or separator function is defined as follows:

$$
f\left(x_{i}\right)=\operatorname{sign}\left(<w, x_{i}>+b\right)
$$

$=\left\{\begin{aligned} 1 & \text { if } y_{i}=1 \\ -1 & \text { if } y_{i}=-1\end{aligned}\right.$

In equation (1), the $w$ parameter represents the weight of inertia, $b$ is the width of the origin point, in which $w \in Z$ and $b \in R$.
Based on the LSVM model, an optimized hyperplane is shown in Figure 2 (34).

In Figure 2, $x_{i}$ is the data points of the two classes labeled $y_{i}=\{1,-1\}$ such that $\langle w, x\rangle+b=0$ represents the optimal hyperplane assigned in the average of the other two lines, that is, $\{x|<w, x\rangle+b=+1\}$ and $\{x \mid<w, x>+b=-1\}$. Also, $w$ denotes a normal vector for the optimal hyperplane, and $b$ is the offset between the hyperplane and the origin plane. Moreover, the margin $M=w / 2$ of the separator is the distance among support vectors. Therefore, the maximum margin can be obtained in the form of the following constraint optimization equation (2):

$$
\text { Minimize : } \frac{1}{2}|w|^{2} \text { subjectto }: y i(w \cdot x+b)-1 \geq 0 \forall \mathrm{i}
$$

Based on the objective function (2), the most common approach for solving an optimization problem is to transform it into a dual problem. First, to get the dual form of the problem, the positive Lagrangian coefficients are multiplied by $\alpha_{i} \geq 0$ and deducted from the objective function, causing in the following equation named a primal problem (Lagrange's initial equation, $L_{p}$ ):

$$
\mathrm{L}_{\mathrm{p}}=\frac{1}{2}|w|^{2}-\sum_{i} \alpha_{\mathrm{i}}\left(\mathrm{y}_{\mathrm{i}}(\mathrm{w} \cdot \mathrm{x}+\mathrm{b})\right)-1
$$

To solve equation (3), we are employed the Karush-KuhnTucker (KKT) conditions, which perform an essential role in constraint optimization problems. These conditions state the necessary and adequate needs for the optimal solution to constraint formulas and must be a derivative of the function regarding the variables equal to zero. Exploiting KKT conditions into $L_{p}$ has been derived from the $L_{p}$ relation to $w$ and $b$, and it sets to zero. So, the equations (4-7) are obtained as follows:

$$
\begin{array}{r}
\frac{\partial}{\partial w_{v}} L_{p}=w_{v}-\sum_{i} \alpha_{i} y_{i} x_{i v}=0 v=1, \ldots, d \\
\frac{\partial}{\partial b} L_{p}=-\sum_{i} \alpha_{i} y_{i}=0 \\
\alpha_{i}\left(y_{i}(w \cdot x+b)\right)-1 \geq 0 i=1, \ldots, l \alpha_{i} \geq 0 \forall_{i} \\
\alpha_{i}\left(y_{i}(w \cdot x+b)\right)-1 \geq 0 \forall_{i}
\end{array}
$$

Consequently, with the assignment of the above formulas into equation (3), equation (8) is gained.

$$
\text { Maximize: } L_{D}=\sum_{i} \alpha_{i}-\frac{1}{2} \sum_{i} \sum_{j} \alpha_{i} \alpha_{j} y_{i} y_{j}\left(x_{i} . x_{j}\right)
$$

Equation (8) is named the dual problem. Hence, $L_{p}$ and $L_{D}$ are both obtained from the same condition. So, the optimal problem can be solved by achieving the minimum $L_{p}$ or the maximum $L_{p}$, the $L_{p}$ double with the condition $\alpha_{i} \geq 0$.

The parameters setting of the used LSVM method is given in Table 2.

The pseudocode of the LSVM is presented below. 
Algorithm 1: The used LSVM method for CAD diagnosis.

Input: Extract the Z-Alizadeh Sani dataset include 303 records and 55 features.

Output: The diagnosed (CAD/normal) class of each test record by the generated LSVM model and determined the evaluation criteria such as accuracy, positive predictive value (PPV), F-measure, sensitivity, specificity, negative predictive value, and AUC

1: Begin

2: Data preprocessing: Transforming nominal records to numerical records and normalizing between zero and one

3: Divide the data using a 10-fold crossvalidation technique

4: $\quad$ Choose the value of the parameters setting based on Table 2

5: While (terminating condition is not happened by 10 fold crossvalidation) do

6: $\quad$ Employ LSVM train for each record

7: $\quad$ Generate LSVM model

8: $\quad$ Employ LSVM classify for testing records

9: $\quad$ End while

10: $\quad$ Return obtaining the evaluation criteria

11: End

\section{Library SVM With RBF}

The SVM is a binary classifier that it can only classify two classes. $\operatorname{LIBSVM}(38,39)$ also supports the multiclass state. The difference between the two-class and multiclass problems regarding training and testing the data is that larger sets or multiclass conditions may be time-consuming. Indeed, the LIBSVM supports four different kernels by default: linear, polynomial, RBF, and sigmoid kernels, so that the RBF is the essential tool for SVM and regression classifications developed by Chavhan et al. (36). The RBF kernel is applied in the training phase (37). The advantage of using the RBF kernel is that it handles the training data to assign specified boundaries. Moreover, the RBF kernel nonlinearly maps samples into a higher-dimensional space. It can handle the training data when the relation between class labels and features is nonlinear. The RBF kernel has fewer numerical difficulties than the polynomial kernel, linear, and sigmoid.

The SVM types are selected through LIBSVM, such as the CSVC and nu-SVC for classification tasks. Also, the epsilon-SVR and nu-SVR are used for regression tasks, and the one-class SVM is performed for distribution estimation. In this paper, the RBF kernel is selected for SVM as formulated in (9).

The most common kernel type is the RBF for SVM.

$$
K\left(x_{i}, x_{j}\right)=\exp \left(-\frac{\left\|x_{i}-x_{j}\right\|^{2}}{2 \sigma^{2}}\right)=\exp \left(-\gamma\left\|x_{i}-x_{j}\right\|^{2}\right)
$$

According to (9), $\sigma$ is the radial of the kernel function. Also, $\gamma=$ represents the kernel parameter. The value of the kernel parameter affects the training rate and the test rate. It should be noted that the efficiency of SVM regarding the accuracy of diagnosis and generalization power is related to the situation of the penalty factor " $c$ " and the kernel parameter " $\gamma$ " (34).

TABLE 2 | The parameters setting of the LSVM method.

\begin{tabular}{ll}
\hline Parameters & Setting \\
\hline Kernel cache & 200 \\
C & 0.1 \\
Convergence epsilon & 0.001 \\
Max iterations & 100,000 \\
L pos & 1.0 \\
L neg & 1.0 \\
Balance cost & $\checkmark$
\end{tabular}

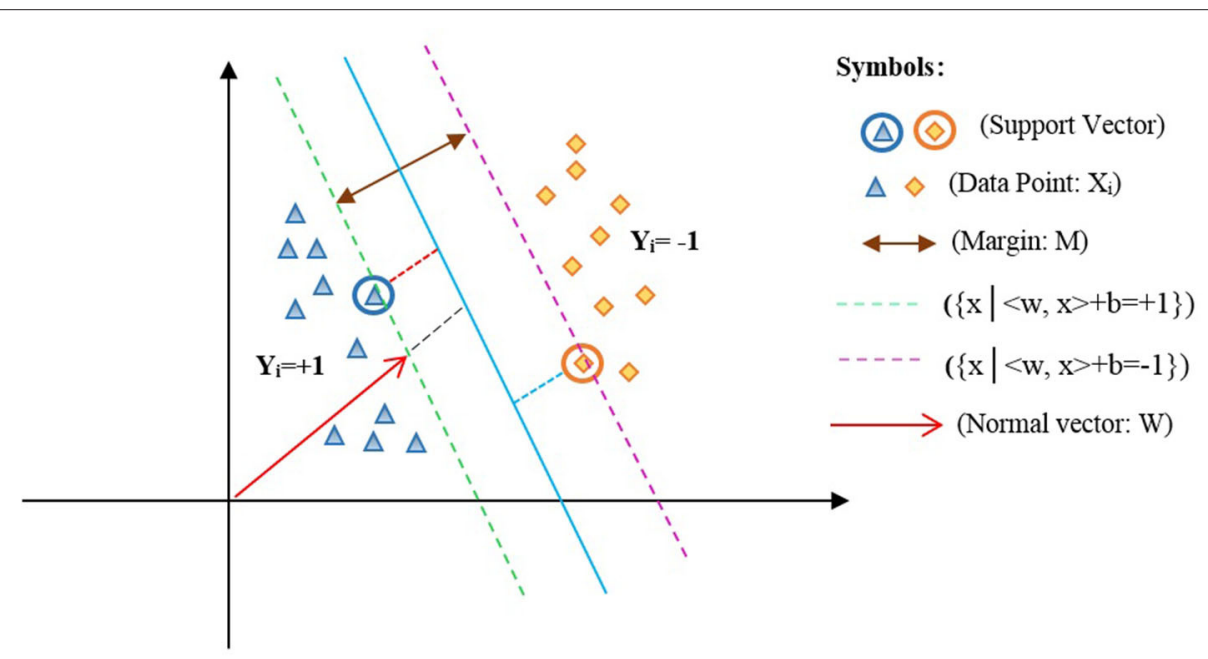

FIGURE 2 | Optimized hyperplane for two-dimensional space. 
Moreover, the C-SVC is used to classify data.

The parameters setting of the used LIBSVM with RBF method is described in Table 3.

The pseudocode of the LIBSVM is given below.

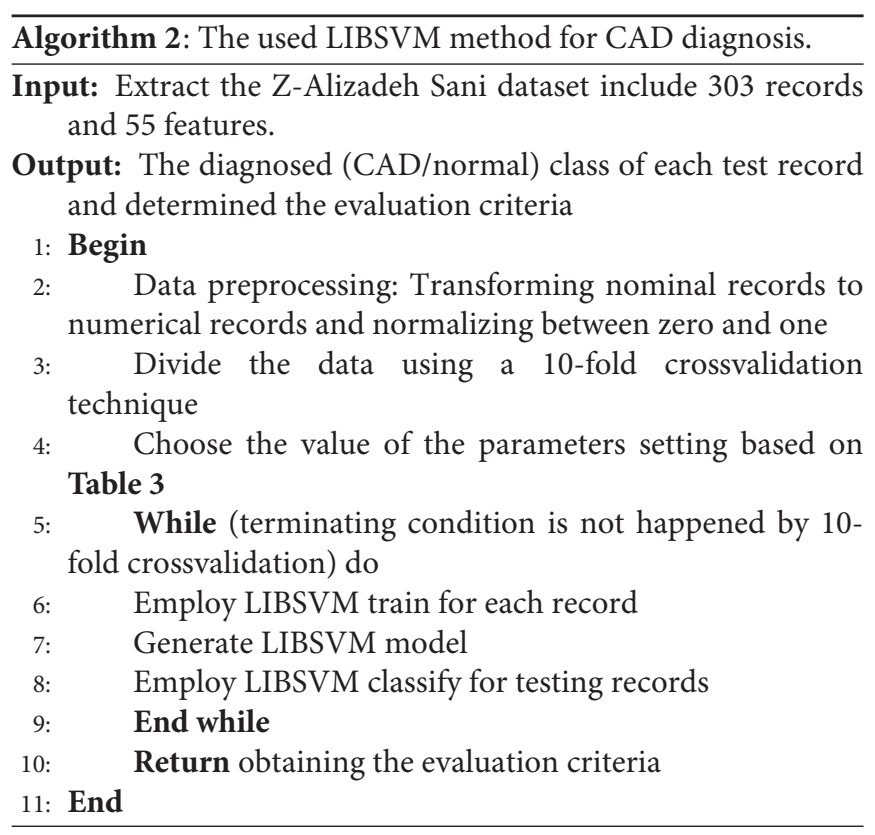

\section{SVM With Analysis of Variance}

In general, kernel types are supported by the SVM such as dot, radial, polynomial, neural, ANOVA, Epachnenikov, Gaussian combination, and multiquadric. The ANOVA kernel is defined by raised to the power " $d$ " of summation of $\exp (-\gamma(x-y))$ where " $\gamma$ " is gamma, and " $d$ " is degree. The " $\gamma$ " and " $d$ " are regulated by the kernel gamma and kernel degree parameters, respectively. Indeed, the ANOVA kernel is also a RBF kernel. It is said to perform well in multidimensional regression and classification problems (40). The ANOVA kernel is formulated as follows:

$$
k(x, y)=\sum_{k=1}^{n} \exp \left(-\sigma\left(x^{k}-\stackrel{k}{y}\right)^{2}\right)^{d}
$$

TABLE 3 | The parameters setting of the LIBSVM method.

\begin{tabular}{ll}
\hline Parameters & Setting \\
\hline SVM type & C-SVC \\
Kernel type & RBF \\
Gamma & 1.0 \\
C & 0.1 \\
Cache size & 80 \\
Epsilon & 0.001 \\
Shrinking & $\checkmark$
\end{tabular}

The parameters setting of the used SVM with the ANOVA method is given in Table 4 .

The pseudocode of the SVM with the ANOVA method is presented below.

Algorithm 3: The used SVM with ANOVA method for CAD diagnosis.

Input: Extract the Z-Alizadeh Sani dataset include 303 records and 55 features.

Output: The diagnosed (CAD/normal) class of each test record and determined the evaluation criteria

1: Begin

2: Data preprocessing: Transforming nominal records to numerical records and normalizing between zero and one

3: Divide the data using a 10-fold cross-validation technique

4: Choose the value of the parameters setting based on Table 4

5: While (terminating condition is not happened by $10-$ fold crossvalidation) do

6: $\quad$ Employ SVM with ANOVA train for each record

7: $\quad$ Generate SVM with ANOVA model

8: $\quad$ Employ SVM with ANOVA classify for testing records

9: $\quad$ End while

10: $\quad$ Return obtaining the evaluation criteria

1: End

\section{Genetic Support Vector Machine Along With ANOVA}

A genetic algorithm is a search heuristic method for solving optimization problems. This optimization algorithm uses heuristic biology techniques such as inheritance and mutation. In a genetic algorithm, to obtain the optimal response, the appropriate generation solutions are combined based on the principle of survival of the most desirable living organisms. In fact, in this algorithm, the solutions to a problem are defined in a chromosome form, consisting of a set of parameters called a gene. So, chromosomes are the proposed solutions to the problem of the genetic algorithm.

TABLE 4 | The parameters setting of the SVM with the ANOVA method.

\begin{tabular}{lc}
\hline Parameters & Setting \\
\hline Kernel type & ANOVA \\
Kernel gamma & 1.0 \\
Kernel degree & 2.0 \\
Kernel cache & 200 \\
C & 0.1 \\
Convergence epsilon & 0.001 \\
Max iterations & 100,000 \\
L pos & 1.0 \\
L neg & 1.0 \\
Balance cost & $\checkmark$
\end{tabular}


The most important application of the genetic algorithm is feature selection. Feature selection can be defined as the process of identifying related features and removing unrelated and duplicate features. The feature selection by the genetic algorithm is caused to the better efficiency of the classification methods. Hence, in this paper, we used a genetic algorithm for subset feature selection.

The stages of the genetic algorithm are as follows:

1) Initial population

The parameter of the initial population specifies the population size, that is, the number of members per generation. The genetic algorithm starts with a set of chromosomes so that several solutions with different combinations of features are randomly generated. Indeed, the chromosomes of the initial population, which are the initial solutions, comprise different combinations of the features. These combinations were randomly incorporated for each chromosome and formed the initial solutions to the problem. Hence, in this paper, using this algorithm, the best subset of the features is selected from the ZAlizadeh Sani dataset (5). These essential features are fed to the SVM classification algorithm to classify the input dataset. In the genetic algorithm process, we set the population size to 50 and set the maximum number of generations to ten. Therefore, the size of each chromosome is related to the number of features, including 55 genes for all features.

\section{2) Determining the fitness function}

The value of each chromosome is determined by the fitness function. This function is used to examine the solutions generated in the initial population. In this paper, the fitness function is equal to accuracy, F-measure, sensitivity, specificity, PPV, and negative predictive value (NPV) (28) as determined in equations (11-16):

$$
\begin{array}{r}
\text { Specificity }=T N / T N+F P \\
\text { Accuracy }=T P+T N / T P+T N+F P+F N \\
\text { Positive Predictive Value }=T P / T P+F P \\
\text { Sensitivity }=T P / T P+F N \\
F-\text { measure }=2 * \frac{\text { precision } * \text { Sensitivity }}{\text { precision }+ \text { Sensitivity }}
\end{array}
$$$$
\text { Negative Predictive Value }=T N / T N+F N
$$

Based on the equations (11-16), the elements of the false positive (FP), false negative (FN), true positive (TP), and true negative $(\mathrm{TN})$ are described as follows:

- FP: The number of samples predicted to be positive is negative.

- FN: The number of samples predicted to be negative is positive.

- TP: The number of samples predicted to be positive is positive.

- TN: The number of samples predicted to be negative is negative.
The performance of the GSVMA method is evaluated using the mentioned fitness functions. Therefore, we tested the generated GSVMA model based on the fitness functions.

\section{3) Selection scheme}

In this stage, based on the fitness criterion, a member of a generation is selected so that members with more compatibility have more probability of making the next generation. The selection schemes such as uniform, roulette wheel (RW), stochastic universal sampling, Boltzmann, rank, tournament, and nondominated sorting exist in the genetic algorithm (6). In this paper, the RW is applied as a select scheme. Based on the scheme, the member with a higher fitness value has more probability of being selected. This scheme is one of the weighted random selection schemes. The probability of choosing each member is obtained according to the equation (17).

$$
P_{i}=\frac{f_{i}}{\sum_{k=1}^{N} f_{k}}
$$

In (17), $P_{i}$ indicates the probability of choosing the member, " $i$ " $f_{i}$ indicates member fitness, " $i$ " and $N$, the number of members in the initial population. In this paper, the initial $P=0.5$. The higher value of $P_{i}$ represents that the probability of choosing the chromosome is high. In other words, this chromosome has a better chance to produce the next generation.

\section{4) The operation of crossover}

After the parent chromosomes are selected by the RW, they must be merged to generate two new children for both parents by the crossover operator. In general, there are three crossover types such as one-point, uniform, and shuffle (6, 41). Using the one-point crossover, a point on two-parent chromosomes is selected and divided into two parts so that one part of the first parent is replaced by one part of the second parent. The other type of crossover is a shuffle that two points on two-parent chromosomes are randomly selected and divided into three parts. Then, one part of the first parent is replaced by one part of the second parent, and the children in three parts are a combination of two parents. The third type of crossover is uniform. Using the uniform crossover, all the chromosome points are selected as the merge points. First, a random number between zero and one for each part of the chromosome is generated. If the generated value is less than a constant value, the genes are moved. In this paper, the shuffle has been selected as the crossover type. Also, crossover " $p$ " is given 0.75 .

\section{5) Mutation action}

After crossover, the mutation action is one of the essential actions to create a new generation. The mutation action is used to modify a member of the current generation to produce a new member. Due to the mutation action by random, the possibility of reaching a better member and 
escaping the local optimization can be efficient. In this paper, the probability value of 1.0 has been considered. This value demonstrates that mutation action is performed to create a new generation.

When the mutation action is performed, the cycle of the genetic algorithm is terminated due to convergence conditions. The convergence condition is determined based on the number of generations (number of generations $=10$ ). Again, construction action of the new generation should be repeated $(6,41)$.

The parameters setting of the used genetic optimization method is given in Table 5.

The pseudocode of the GSVMA method is presented below.

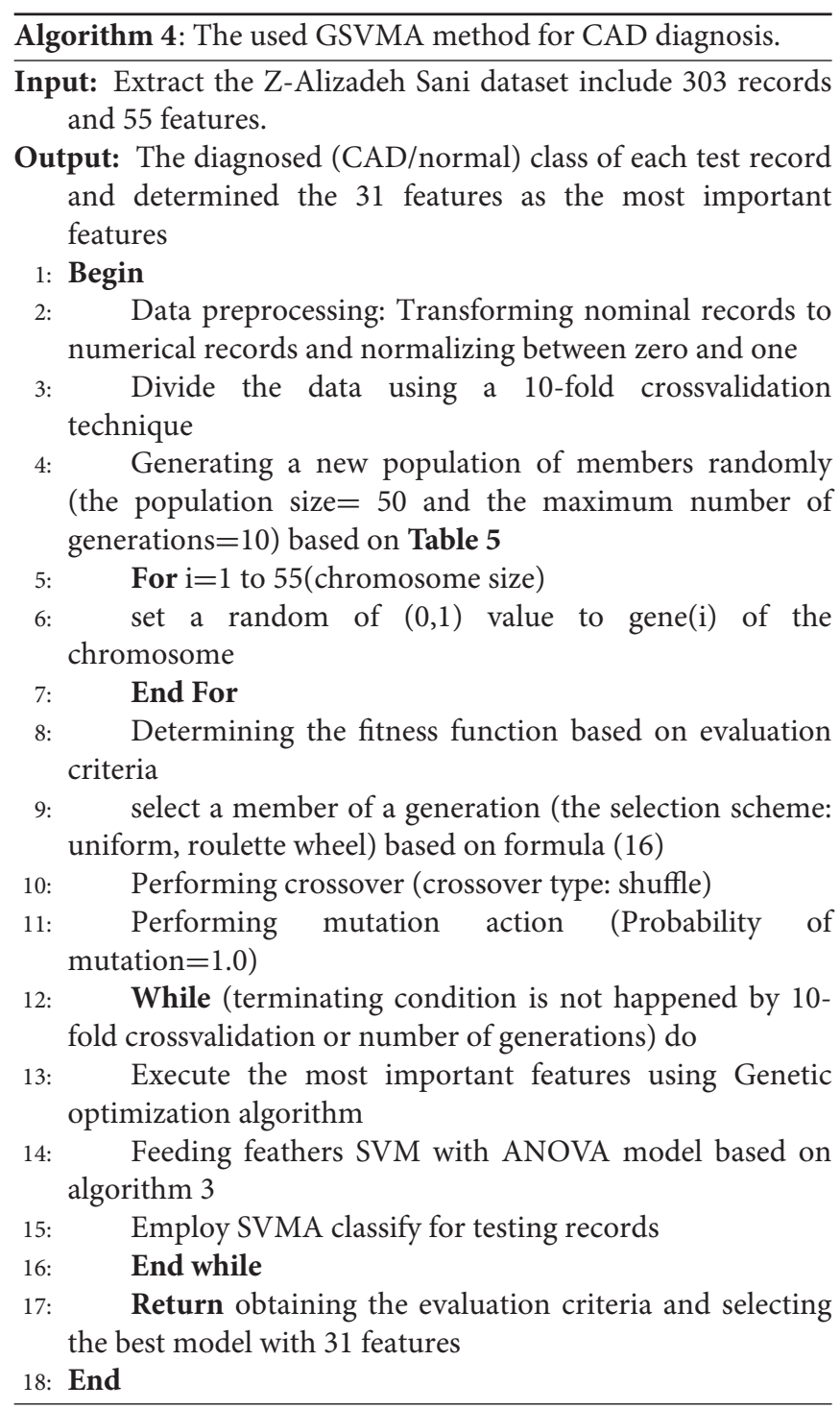

Based on algorithm 4, the genetic optimization method has been applied for feature selection, and the SVM with the ANOVA model has been used for classifying the dataset.
TABLE 5 | The parameters setting of the genetic optimization algorithm.

\begin{tabular}{ll}
\hline Parameters & Setting \\
\hline Population size & 50 \\
Maximum number of generations & 10 \\
Normalize weights & $\checkmark$ \\
Plot generations & 10 \\
Draw dominated points & $\checkmark$ \\
Initial probability & 0.5 \\
Size of each chromosome = total of features & 55 \\
Probability of mutation & 1.0 \\
Crossover probability & 0.75 \\
Crossover type & Shuffle \\
Maximum fitness & Infinity \\
Fitness function & Accuracy, PPV, \\
& F-measure, sensitivity, \\
& specificity, and NPV
\end{tabular}

TABLE 6 | Confusion matrix for diagnosis of CAD.

\begin{tabular}{lll}
\hline The predicted class & \multicolumn{2}{c}{ The actual class } \\
\cline { 2 - 3 } & CAD & Normal \\
\hline Positive & True positive & False positive \\
Negative & False negative & True negative \\
\hline
\end{tabular}

\section{RESULTS}

In this section, the evaluation results for the classification methods are obtained. These methods are the SVM with ANOVA, LSVM, LIBSVM with RBF, and GSVMA. Based on Table 6, accuracy (ACC), PPV, F-measure, sensitivity, specificity, NPV, and AUC had been achieved by the confusion matrix. In this paper, we have used RapidMiner Studio version 9.9 to implement the methods in the CAD diagnosis and classification process.

The evaluation criteria of the methods were obtained based on equations (11-16) (28).

By comparing the performance of the methods, the ACC rates of the SVM with ANOVA, LSVM, and LIBSVM with RBF were achieved $85.13,86.11$, and $84.78 \%$, respectively, whereas the ACC rate of the GSVMA method is $89.45 \%$ based on the 10-FCV technique. According to the other criteria, the GSVMA method had the highest PPV, F-measure, sensitivity, and specificity. Moreover, another crucial criterion used to determine the classification methods is the AUC criterion. The AUC indicates the measure of the area under the Receiver Operating Characteristic (ROC). In other words, the AUC transforms the ROC curve into a numeric demonstration of performance for classification models. The AUC of the GSVMA method is obtained $100 \%$. The results of the evaluation criteria for methods through the 10-FCV are indicated in Table 7. Also, the ROC curve was illustrated for the GSVMA method in Figure 3.

Based on Figure 3, the ROC diagram for the GSVMA method demonstrates that the AUC value $=100 \%$. 
TABLE 7 | The comparison of the methods based on the Z-Alizadeh Sani dataset in this study.

\begin{tabular}{|c|c|c|c|c|c|c|c|}
\hline Methods & $\mathrm{ACC}(\%)$ & PPV(\%) & F-measure (\%) & Sensitivity (\%) & Specificity(\%) & NPV(\%) & AUC(\%) \\
\hline SVM with ANOVA & 85.13 & 80.24 & 72.01 & 69.19 & 91.62 & 68.97 & 89 \\
\hline Linear SVM & 86.11 & 77.21 & 75.55 & 74.75 & 90.71 & 74.71 & 92.4 \\
\hline LIBSVM with RBF & 84.78 & 76.24 & 72.38 & 70.03 & 90.74 & 70.11 & 82.1 \\
\hline GSVMA & 89.45 & 100 & 80.49 & 81.22 & 100 & 92.9 & 100 \\
\hline
\end{tabular}

Bold the best values of the proposed method (GSVMA) to show our method is the best.

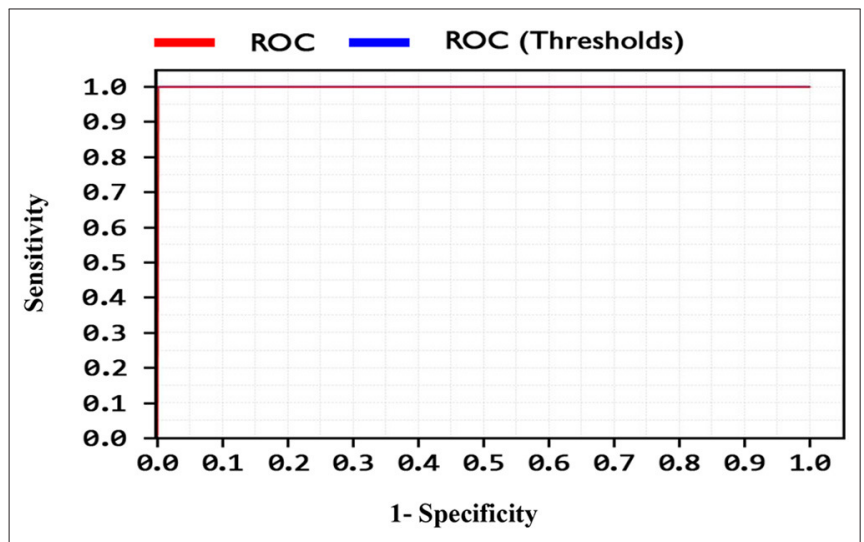

FIGURE 3 | ROC curve for GSVMA method.

Also, the criteria of the ACC, F-measure, PPV, sensitivity, specificity, and NPV are illustrated in Figures 4-9, respectively. Moreover, in the 10-FCV technique, the GSVMA method was trained for 10 generations on the Z-Alizadeh Sani dataset. To find the optimal response of the evaluation criteria (fitness function $=$ criteria), a set of initial responses are generated in each generation so that the set of responses converges toward the optimal response. In this study, convergence is related to the tenth generation. Therefore, the mentioned criteria were obtained based on the best generation (generation 10).

According to Figure 4, the ACC rate has obtained more than $88 \%(89.45 \%)$ through the GSVMA method for generation 10 .

Based on Figure 5, the performance of the GSVMA method achieved F-measure of $80.49 \%$ for generation 10 .

Figure 6 shows that the PPV has reached a maximum of $100 \%$ for the tenth generation.

By observing Figure 7, it can be inferred that the sensitivity has been more than $80 \%$ (81.22) using the GSVMA method for the tenth generation.

Regarding the specificity criterion, Figure 8 shows the maximum value of $100 \%$ using the GSVMA method for the tenth generation.

Another crucial evaluation criterion is the NPV in clinical practice. The NPV is the probability that subjects with a negative test rightly have healthy. The NPV was obtained on average 92.9\% as shown in Figure 9. Similarly, three methods such as LIBSVM with RBF, LSVM, and SVM with ANOVA have been applied to the Z-Alizadeh Sani dataset. We presented the results

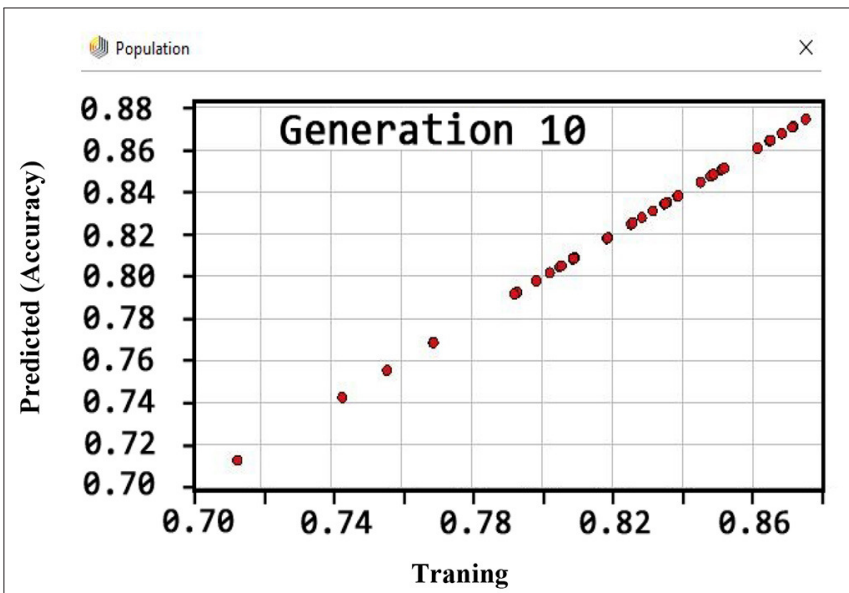

FIGURE 4 | ACC diagram for GSVMA method for generation 10.

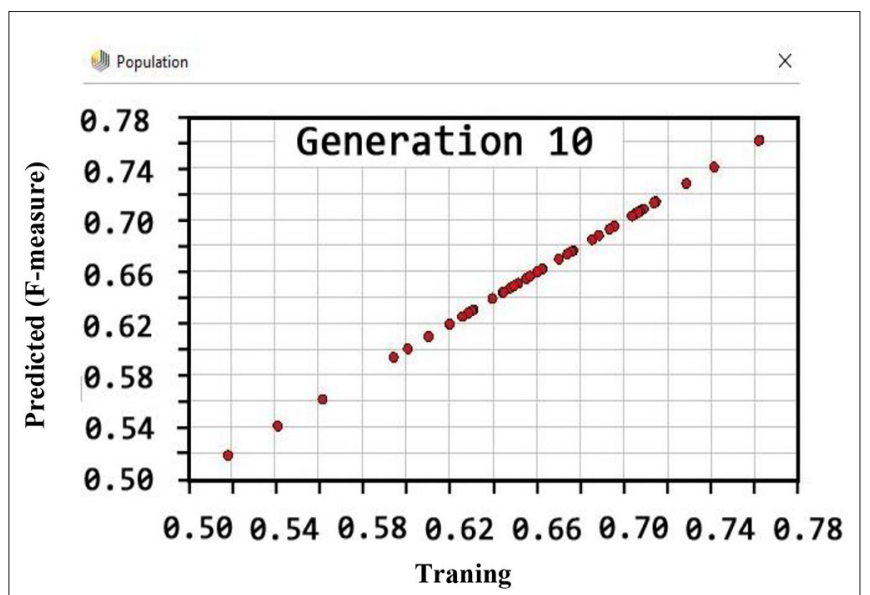

FIGURE 5 | F-measure diagram for GSVMA method for generation 10.

of these methods in Table 7. Based on Table 7, the accuracy of the LSVM, SVM with ANOVA, and LIBSVM with RBF is 86.11, 85.13 , and $84.78 \%$, respectively. The PPV for the SVM with ANOVA, LSVM, and LIBSVM with RBF models is obtained as $80.24,77.21$, and 76.24 , respectively. In terms of the F-measure, sensitivity, specificity, and NPV criteria, the LSVM method has a better performance compared to the other two methods.

In addition, one of the important criteria of the evaluation of methods is the AUC criterion. The AUC of methods was 


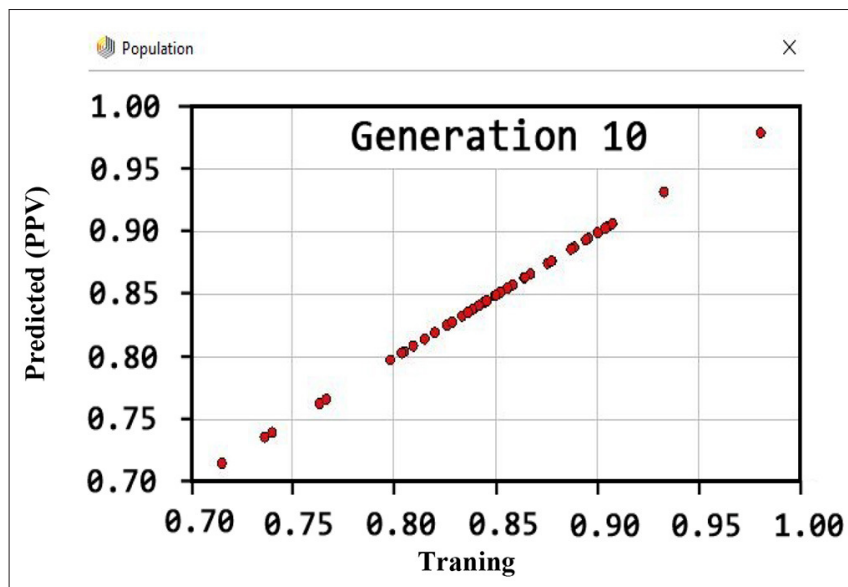

FIGURE 6 | PPV diagram for GSVNA method for generation 10.

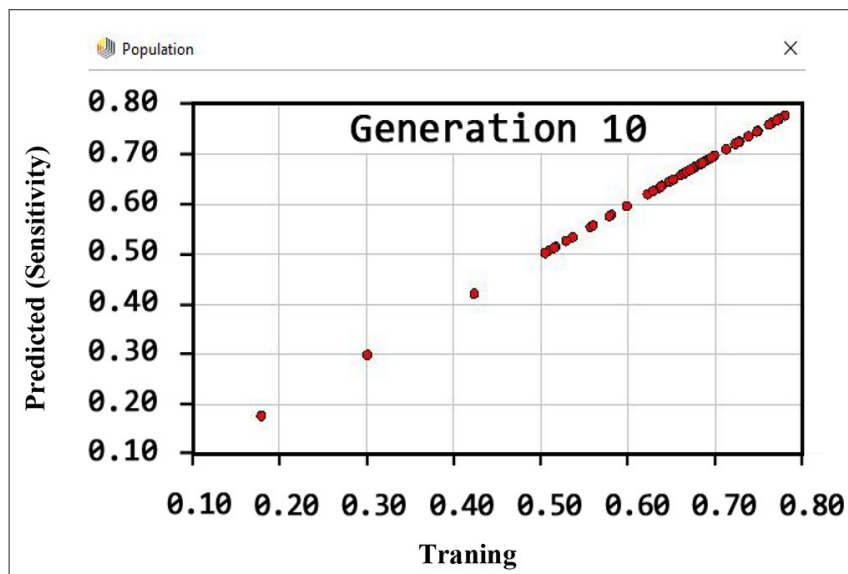

FIGURE 7 | Sensitivity diagram for GSVMA method for generation 10.

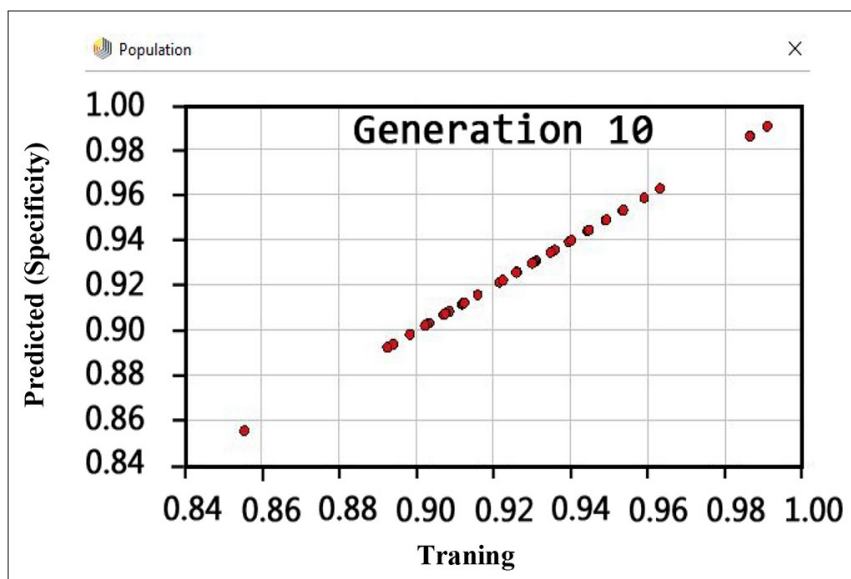

FIGURE 8 | Specificity diagram for GSVNA method for generation 10.

achieved as 92.4, 89, and 82.1\% for LSVM, SVM with ANOVA, and LIBSVM with RBF methods, respectively. The ROC curve for

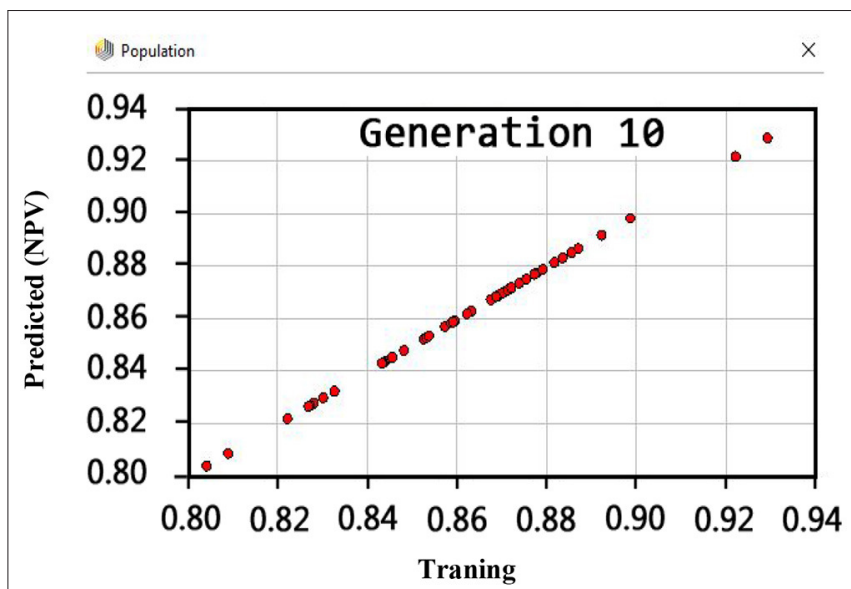

FIGURE 9 | NPV diagram for GSVNA method for generation 10.

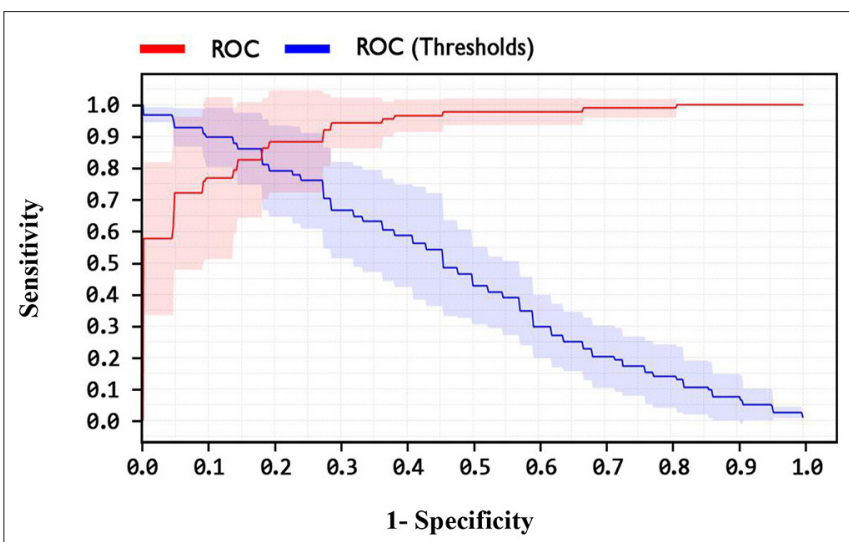

FIGURE 10 | ROC curve for LSVM method.

the LSVM, SVM with ANOVA, and LIBSVM with RBF methods is shown in Figures 10-12, respectively.

By observing the Figures 10-12, it can be concluded that the LSVM method has better performance with an AUC of $92.4 \%$ than SVM with ANOVA and LIBSVM with RBF methods.

Overall, the proposed GSVMA method has the best performance compared to the other methods in terms of accuracy, F-measure, PPV, NPV, sensitivity, specificity, and AUC. Figure 13 shows the comparison between the methods based on the seven criteria.

Moreover, according to the proposed method, out of 35 features, 31 features were selected using the genetic optimization algorithm. The crucial features include sex, CRF, CVA, airway disease, thyroid disease, $\mathrm{CHF}$, systolic murmur, diastolic murmur, low TH ang, LVH, poor R progression, VHD, age, HTN, exsmoker, FH, PR, typical chest pain, function class, Q wave, St elevation, T inversion, FBS, TG, LDL, ESR, lymph, Neut, PLT, EF-TTE, and region RWMA. 


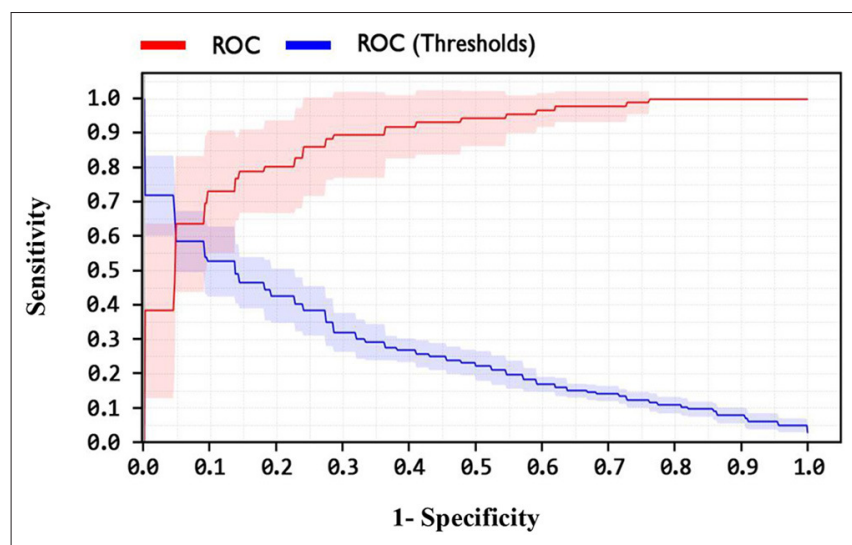

FIGURE 11 | ROC curve for SVM with ANOVA method.

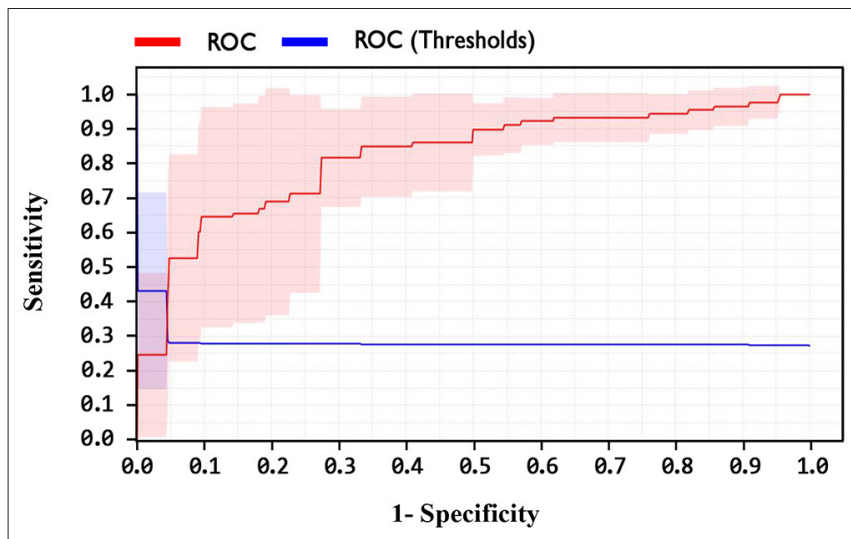

FIGURE 12 | ROC curve for LIBSVM with RBF method.

\section{DISCUSSION}

In this paper, we demonstrate the accuracy of $89.45 \%$ using the proposed GSVMA method for CAD diagnosis on the Z-Alizadeh Sani dataset by identifying 31 features. Finally, we compared our proposed method with the work of other researchers based on the Z-Alizadeh Sani dataset, as demonstrated in Table 8.

Based on Table 8, in the Qin et al. study (8), several feature selection methods had been implemented on the Z-Alizadeh Sani CHD dataset. The various assessment criteria to evaluate features coupled with a heuristic search strategy and seven classification methods are utilized. They further proposed an ensemble algorithm based on multiple feature selection (EAMFS). The proposed EA-MFS method had better results with a reported accuracy of 93.70 and $95.53 \%$ F1-measure. In Cüvitoglu and Işik's study (10), an ensemble learner based on the combination of naïve Bayes, random forest, SVM, artificial neural networks (ANNs), and k-nearest neighbor algorithm is developed to diagnose CAD. Also, each of these methods is applied to the dataset separately. The authors performed a $t$-test for feature selection and reduced the feature space from 54 to 25. Moreover, they implemented PCA to reduce dimensionality further. Between the six methods, the best performance of the ANN achieved an AUC of 93\%. Kiliç and Keleş (12) attempted to select the most convenient features to achieve better performance. They have used the artificial bee colony method on the Z-Alizadeh Sani dataset. The results showed that 16 of 56 features are more meaningful to predict CAD. They reported that a higher accuracy was achieved employing the selected features. Abdar et al. (14) proposed a model combining several traditional ML methods using ensemble learning techniques titled nested ensemble nu-support vector classification (NE-nu-SVC) to predict CAD. Also, they employed a feature selection routine based on a genetic algorithm and a filtering method to adjust data. The reported that accuracy of the NE-nu-SVC method is $94.66 \%$ for Z-Alizadeh Sani and $98.60 \%$ for Cleveland CAD datasets. In (16), Abdar et al. introduced the N2Genetic optimizer, which is a genetic-based algorithm and particle swarm optimization. Using the N2Genetic-nuSVM proposed, they achieved an accuracy of $93.08 \%$ and F1-score of $91.51 \%$ in the Z-Alizadeh Sani dataset for predicting CAD. In another study, Kolukisa et al. (17) examined two feature selection approaches to extract the most convenient set of features for the Z-Alizadeh Sani dataset. First, the features were selected based on medical doctor recommendations. According to clinically significant findings and Framingham heart study risk factors labeled features. The second method of feature selection was reported to improve the performance of ML algorithms. A combination of three ensemble learners, random forest, gradient boosting machine, and extreme gradient boosting, form a classifier to predict coronary heart disease in the work of Tama et al. (19). Moreover, a particle swarm optimization-based feature selection model takes the most valuable data features to feed the classifier efficiently. The functionality of the proposed system is verified by having Z-Alizadeh Sani, Statlog, Cleveland, and Hungarian datasets as the input data. The authors claim that the performance of their proposed model outperforms the present methods established on traditional classifier ensembles. They report a $98.13 \%$ accuracy, 96.60\% F1-score, and 0.98 AUC to classify the Z-Alizadeh Sani dataset. The effectiveness of ANN and adaptive boosting algorithms to predict CAD was tested in the work of Terrada et al. (20). Data for this study were collected from Z-Alizadeh Sani, Hungarian, UCI repository, and Cleveland datasets, and 17 features were manually selected based on the atherosclerosis risk factors. The results indicated that ANNs show more promising performance over the adaptive boosting method. In (22), a hybrid algorithm based on emotional neural networks (EmNNs) and particle swarm optimization (PSO) is proposed by Shahid and Singh for CAD diagnosis. In addition, they implemented four unique feature selection techniques on the ZAlizadeh Sani dataset to boost the functionality of the proposed model. Generally, their method has a better performance than the PSO-ANFIS model. The F1-score, accuracy, sensitivity, specificity, and PPV of the model are 92.12, 88.34, 91.85, 78.98, and $92.37 \%$, respectively. According to Ghiasi et al. (23), only 40 independent parameters of the Z-Alizadeh Sani dataset affect the diagnosis of CAD. The authors apply the classification and regression tree (CART) method for this purpose. They further developed three additional CARD models utilizing 5, 


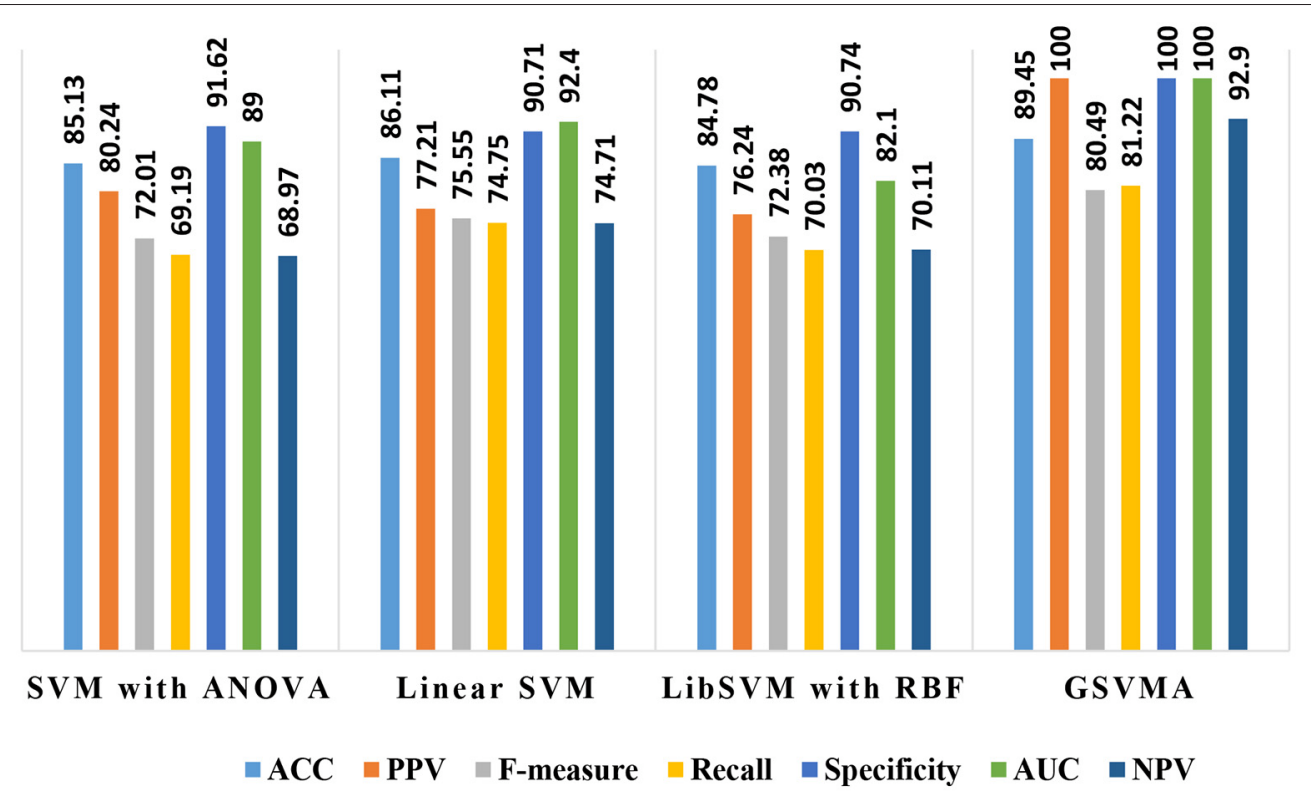

FIGURE 13 | A comparison between the performance of methods based on the seven criteria.

TABLE 8 | Comparison between the proposed GSVMA method and the work of other researchers based on the original Z-Alizadeh Sani dataset.

\begin{tabular}{|c|c|c|c|c|c|}
\hline References & Techniques & $\begin{array}{l}\text { No. of } \\
\text { crossvalidation }\end{array}$ & No. of features & ACC (\%) & AUC (\%) \\
\hline Qin et al. (8) & $\begin{array}{l}\text { Ensemble algorithm-multiple } \\
\text { feature selection, (EA-MFS) }\end{array}$ & 10-FCV & 34 & $93.70+-0.48 \%$ & $\mathrm{NC}$ \\
\hline Cüvitoglu et al. (10) & Artificial neural network & 10-FCV & 25 & 87.85 & 93 \\
\hline Kiliç et al. (12) & Artificial Bee colony & - & 16 & 89.4 & $\mathrm{NC}$ \\
\hline Abdar et al. (14) & NE-nu-SVC & 10-FCV & 16 & 94.66 & $\mathrm{NC}$ \\
\hline Abdar et al. (16) & N2Genetic-nuSVM & 10-FCV & 29 & 93.08 & $\mathrm{NC}$ \\
\hline Kolukisa et al. (17) & $\begin{array}{l}\text { Ensemble classifier with } \\
\text { Fisher linear discriminant } \\
\text { analysis }\end{array}$ & $5,10,20-F C V$ & 55 & 92.07 & 95.3 \\
\hline Tama et al. (19) & $\begin{array}{l}\text { Two-tier ensemble particle } \\
\text { swarm optimization(PSO)- } \\
\text { based feature } \\
\text { selection }\end{array}$ & 10-FCV & 27 & 98.13 & 98.7 \\
\hline Terrada et al. (20) & $(\mathrm{ANN})$ & - & 17 & 94 & 94 \\
\hline Shahid et al. (22) & Hybrid PSO-EmNN & 10-FCV & 22 & 88.34 & $\mathrm{NC}$ \\
\hline Ghiasi et al. (23) & CART & 10-FCV & 5 & 92.41 & $\mathrm{NC}$ \\
\hline Dahal et al. (24) & SVM & 10-FCV & 15 & 89.47 & 88.68 \\
\hline Velusamy et al. (25) & $\begin{array}{l}\text { Weighted-average Voting } \\
\text { ensemble (WAVEn) }\end{array}$ & 10-FCV & 5 & 98.97 & $\mathrm{NC}$ \\
\hline Hassannataj et al. (5) & Random trees & 10-FCV & 40 & 91.47 & 96.7 \\
\hline The Proposed Method & GSVMA & 10-FCV & 31 & 89.45 & 100 \\
\hline
\end{tabular}

NC, Not considered.

10 , and 18 selected features. For the developed model with five features, the reported accuracy is $92.41 \%$. Also, a $77.01 \%$ true negative rate and $98.61 \%$ true positive value are reported for the model.

Dahal et al. (24) performed logistic regression, random forest, SVM, and K-nearest neighbors algorithms for CAD detection on the Z-Alizadeh Sani dataset to determine the most efficient technique. The results indicate that SVM has a better performance over other tested methods with $89.47 \%$ of accuracy. In (25), a study of CAD diagnosis was conducted using the weighted-average voting ensemble (WAVEn) method. Using this method, an accuracy of $98.97 \%$ was obtained on five features. 
Hassannataj et al. (5) used the random trees (RTs) on the 303 samples with 55 features. They have compared the RTs model with SVM, the C5.0 decision tree, and the CHAID decision tree. As a result, using the RTs model, 40 features were ranked with an accuracy of $91.47 \%$, which RTs model has the best performance compared to the other models.

The results demonstrate the robustness of our proposed method in the diagnosis and prediction of CAD. Applying the GSVMA method, an accuracy of $89.45 \%$ was obtained by identifying 31 features. To the best of our knowledge, using this method, the AUC was achieved $100 \%$ on the original ZAlizadeh Sani for the first time. Also, no previous works in the literature have investigated the NPV in CAD diagnosis, so that this criterion is essential in clinical practice. Despite these advances in the diagnosis of heart disease, there are limitations to the diagnosis process that we list below.

- Need a larger dataset to apply to the proposed GSVMA method.

- Lack of access to the real laboratory environment to record people's data in electronic profiles.

- Requiring the interaction of physicians and researchers to evaluate the results obtained properly.

\section{CONCLUSIONS}

In this study, a hybrid method, namely GSVMA, is proposed to help the effective diagnosis and prediction of CAD by selecting essential features. This method was evaluated on the ZAlizadeh Sani dataset. The GSVMA method consists of two main blocks. The first is the genetic optimization algorithm, in which essential features are selected by this algorithm. The second is the SVM algorithm with ANOVA kernel, which is used for classifying the input dataset. We carried out data preprocessing by converting nominal data to numerical data and performing a range transformation technique. Also, the 10-fold crossvalidation technique is used to split the dataset into two groups: $90 \%$ for

\section{REFERENCES}

1. Martin-Isla C, Campello VM, Izquierdo C, Raisi-Estabragh Z, Baeßler B, Petersen SE, et al. Image-based cardiac diagnosis with machine learning: a review. Front Cardiovasc Med. (2020) 7:1. doi: 10.3389/fcvm.2020.00001

2. Bagula AB, Djenouri D, Karbab E. On the relevance of using interference and service differentiation routing in the internet-of-things. In: Internet of Things, Smart Spaces, and Next Generation Networking. Berlin; Heidelberg: Springer (2013). p. 25-35. doi: 10.1007/978-3-642-40316-3_3

3. Hampe N, Wolterink JM, Van Velzen SG, Leiner T, Išgum I. Machine learning for assessment of coronary artery disease in cardiac CT: a survey. Front Cardiovasc Med. (2019) 6:172. doi: 10.3389/fcvm.2019.00172

4. Lilly LS, Braunwald E. Braunwald's Heart Disease: A Textbook of Cardiovascular Medicine. Philadelphia, PA: Elsevier Health Sciences (2012).

5. Joloudari JH, Hassannataj Joloudari E, Saadatfar H, Ghasemigol M, Razavi SM, Mosavi A, et al. Coronary artery disease diagnosis; ranking the significant features using a random trees model. Int J Environ Res Public Health. (2020)731:17. doi: 10.3390/ijerph17030731

6. Arabasadi Z, Alizadehsani R, Roshanzamir M, Moosaei H, Yarifard AA. Computer aided decision making for heart disease detection using hybrid training and $10 \%$ for testing. Moreover, other methods such as SVM with ANOVA, LSVM, and LIBSVM with RBF have been utilized to diagnose CAD. The proposed GSVMA method had the best performance compared to the mentioned methods regarding the accuracy of $89.45 \%$, a PPV of $100 \%$, a F-measure of $80.49 \%$, a specificity of $100 \%$, a sensitivity of $81.22 \%$, a NPV of $92.9 \%$, and an AUC of $100 \%$, on 31 features among 55 features. By comparing the proposed method with related works, we found that the GSVMA method has good accuracy and AUC rates with the essential features. Besides, no previous works have studied the NPV in CAD diagnosis. In future work, if a larger dataset is available, the GSVMA method could be utilized. In addition, metaheuristic methods that include tabu search, iterated local search, simulated annealing, and variable neighborhood search can be used for feature selection. Then, each of these methods can be combined with machine learning methods.

\section{DATA AVAILABILITY STATEMENT}

The datasets presented in this study can be found in online repositories. The names of the repository/repositories and accession number(s) can be found at: https://archive.ics.uci.edu/ $\mathrm{ml} /$ datasets/Z-Alizadeh+Sani, 20403598.

\section{AUTHOR CONTRIBUTIONS}

$\mathrm{JH}, \mathrm{FA}$, and RA designed the study. JH, FA, MN, and RA wrote the paper. JH, FA, MN, RA, and AM edited the paper. JH carried out the software on the Z-Alizadeh Sani dataset. JH, FA, RA, and IN generated all figures and tables. All authors have read and approved the final version of the paper.

\section{FUNDING}

This work was supported by Alexander von Humboldt Foundation under project AvH0019272. neural network-Genetic algorithm. Comput Methods Prog Biomed. (2017) 141:19-26.. doi: 10.1016/j.cmpb.2017.01.004

7. Alizadehsani R, Habibi J, Hosseini MJ, Mashayekhi H, Boghrati R, Ghandeharioun A, et al. A data mining approach for diagnosis of coronary artery disease. Comput Methods Prog Biomed. (2013) 111:5261. doi: 10.1016/j.cmpb.2013.03.004

8. Qin C-J, Guan Q, Wang X-P. Application of ensemble algorithm integrating multiple criteria feature selection in coronary heart disease detection. Biomed Eng Appl Basis Commun. (2017) 29:1750043. doi: $10.4015 /$ S1016237217500430

9. Alizadehsani R, Zangooei MH, Hosseini MJ, Habibi J, Khosravi A, Roshanzamir $\mathrm{M}$, et al. Coronary artery disease detection using computational intelligence methods. Knowled Based Syst. (2016) 109:187-97. doi: 10.1016/j.knosys.2016.07.004

10. Cüvitoglu A, I sik Z. Classification of CAD dataset by using principal component analysis and machine learning approaches. In: 2018 5th International Conference on Electrical and Electronic Engineering (ICEEE). Istanbul: IEEE (2018). p. 340-3. doi: 10.1109/ICEEE2.2018.8391358

11. Alizadehsani R, Hosseini MJ, Sani ZA, Ghandeharioun A, Boghrati R. Diagnosis of coronary artery disease using cost-sensitive algorithms. In: 2012 
IEEE 12th International Conference on Data Mining Workshops. Brussels: IEEE (2012). p. 9-16. doi: 10.1109/ICDMW.2012.29

12. Kiliç Ü, Keleş MK. Feature selection with artificial bee colony algorithm on Z-Alizadeh Sani dataset. In: 2018 Innovations in Intelligent Systems and Applications Conference (ASYU). Adana: IEEE (2018). p. 1-3. doi: 10.1109/ASYU.2018.8554004

13. Alizadehsani R, Hosseini MJ, Khosravi A, Khozeimeh F, Roshanzamir $\mathrm{M}$, Sarrafzadegan $\mathrm{N}$, et al. Non-invasive detection of coronary artery disease in high-risk patients based on the stenosis prediction of separate coronary arteries. Comput Methods Prog Biomed. (2018) 162:119-27. doi: 10.1016/j.cmpb.2018.05.009

14. Abdar M, Acharya UR, Sarrafzadegan N, Makarenkov V. NE-nu-SVC: a new nested ensemble clinical decision support system for effective diagnosis of coronary artery disease. IEEE Access. (2019) 7:16760520. doi: 10.1109/ACCESS.2019.2953920

15. Alizadehsani R, Habibi J, Sani ZA, Mashayekhi H, Boghrati R, Ghandeharioun A, et al. Diagnosing coronary artery disease via data mining algorithms by considering laboratory and echocardiography features. Res Cardiovasc Med. (2013) 2:133. doi: 10.5812/cardiovascmed.10888

16. Abdar M, Ksiazek W, Acharya UR, Tan R-S, Makarenkov V, Pławiak P. A new machine learning technique for an accurate diagnosis of coronary artery disease. Comput Methods Prog Biomed. (2019) 179:104992. doi: 10.1016/j.cmpb.2019.104992

17. Kolukisa B, Hacilar H, Kuş M, Bakir-Güngör B, Aral A, Güngör VÇ. Diagnosis of coronary heart disease via classification algorithms and a new feature selection methodology. Int J Data Mining Sci. (2019) 1:8-15.

18. Nasarian E, Abdar M, Fahami MA, Alizadehsani R, Hussain S, Basiri ME, et al. Association between work-related features and coronary artery disease: A heterogeneous hybrid feature selection integrated with balancing approach. Pattern Recogn Lett. (2020) 133:33-40. doi: 10.1016/j.patrec.2020.02.010

19. Tama BA, Im S, Lee S. Improving an intelligent detection system for coronary heart disease using a two-tier classifier ensemble. BioMed Research International. (2020) 2020:9816142. doi: 10.1155/2020/9816142

20. Terrada O, Cherradi B, Hamida S, Raihani A, Moujahid H, Bouattane O. Prediction of patients with heart disease using artificial neural network and adaptive boosting techniques. In: 2020 3rd International Conference on Advanced Communication Technologies and Networking (CommNet). Marrakech: IEEE (2020). p. 1-6. doi: 10.1109/CommNet49926.2020.9199620

21. Shahid AH, Singh MP, Roy B, Aadarsh A. Coronary artery disease diagnosis using feature selection based hybrid extreme learning machine. In: 2020 3rd International Conference on Information and Computer Technologies (ICICT). San Jose, CA: IEEE (2020). p. 341-6. doi: 10.1109/ICICT50521.2020.00060

22. Shahid AH, Singh MP. A novel approach for coronary artery disease diagnosis using hybrid particle swarm optimization based emotional neural network. Biocybernet Biomed Eng. (2020) 40:1568-85. doi: 10.1016/j.bbe.2020. 09.005

23. Ghiasi MM, Zendehboudi S, Mohsenipour AA. Decision tree-based diagnosis of coronary artery disease: CART model. Comput Methods Prog Biomed. (2020) 192:105400. doi: 10.1016/j.cmpb.2020.105400

24. Dahal KR, Gautam Y. Argumentative comparative analysis of machine learning on coronary artery disease. Open J Stat. (2020) 10:694-705. doi: 10.4236/ojs.2020.104043

25. Velusamy D, Ramasamy K. Ensemble of heterogeneous classifiers for diagnosis and prediction of coronary artery disease with reduced feature subset. Comput Methods Prog Biomed. (2021) 198:105770. doi: 10.1016/j.cmpb.2020.105770

26. Pławiak P. Novel genetic ensembles of classifiers applied to myocardium dysfunction recognition based on ECG signals. Swarm Evolution Comput. (2018) 39:192-208. doi: 10.1016/j.swevo.2017.10.002

27. Alizadehsani R, Roshanzamir M, Abdar M, Beykikhoshk A, Khosravi A, Panahiazar $\mathrm{M}$, et al. A database for using machine learning and data mining techniques for coronary artery disease diagnosis. Sci Data. (2019) 6:227. doi: 10.1038/s41597-019-0206-3
28. Joloudari JH, Saadatfar H, Dehzangi A, Shamshirband S. Computeraided decision-making for predicting liver disease using PSO-based optimized SVM with feature selection. Informat Med Unlocked. (2019) 17:100255. doi: 10.1016/j.imu.2019.100255

29. Joloudari JH, Haderbadi M, Mashmool A, GhasemiGol M, Band SS, Mosavi A. Early detection of the advanced persistent threat attack using performance analysis of deep learning. IEEE Access. (2020) 8:18612537. doi: 10.1109/ACCESS.2020.3029202

30. Boser BE, Guyon IM, Vapnik VN, editors. A training algorithm for optimal margin classifiers. In: Proceedings of the Fifth Annual Workshop on Computational Learning Theory. Pittsburgh Pennsylvania. ACM (1992). p. 144-52. doi: 10.1145/130385.130401

31. Cortes C, Vapnik V. Support-vector networks. Machine Learn. (1995) 20:27397. doi: 10.1007/BF00994018

32. Cristianini N, Shawe-Taylor J. An Introduction to Support Vector Machines and Other Kernel-Based Learning Methods. Cambridge University Press (2000).

33. Chang Y-W, Hsieh C-J, Chang K-W, Ringgaard M, Lin C-J. Training and testing low-degree polynomial data mappings via linear SVM. J Machine Learn Res. (2010) 11:1471-90. doi: 10.5555/1756006.1859899

34. Shamshirband S, Joloudari JH, GhasemiGol M, Saadatfar H, Mosavi A, Nabipour N. FCS-MBFLEACH: designing an energy-aware fault detection system for mobile wireless sensor networks. Mathematics. (2020) 8:28. doi: $10.3390 /$ math 8010028

35. Sharifrazi D, Alizadehsani R, Roshanzamir M, Joloudari JH, Shoeibi A, Jafari $M$, et al. Fusion of convolution neural network, support vector machine and Sobel filter for accurate detection of COVID19 patients using X-ray images. Biomed Signal Process Control. (2021) 68:102622. doi: 10.1016/j.bspc.2021.102622

36. Chavhan YD, Yelure BS, Tayade KN. Speech emotion recognition using RBF kernel of LIBSVM. In: 2015 2nd International Conference on Electronics and Communication Systems (ICECS). Coimbatore: IEEE (2015). p. 1132-35. doi: 10.1109/ECS.2015.7124760

37. Souza CR. Kernel functions for machine learning applications. Creative Commons Attribut Noncommercial Share Alike. (2010) 3:29.

38. Wankhade SB, Tijare P, Chavhan Y. Speech emotion recognition system using SVM AND LIBSVM. Int J Comput Sci Applications. (2011) 4.

39. Chang C-C, Lin C-J. LIBSVM: a library for support vector machines. ACM Transac Intel Syst Technol. (2011) 2:1-27. doi: 10.1145/1961189.1961199

40. Hofmann T, Schölkopf B, Smola AJ. Kernel methods in machine learning. Ann Stat. (2008) 36:1171-220. doi: 10.1214/009053607000000677

41. Kaya Y, Uyar M. A novel crossover operator for genetic algorithms: ring crossover. arXiv [Preprint]. (2011). arXiv: 11050355. Available online at: https://arxiv.org/abs/1105.0355 (accessed January 11, 2021).

Conflict of Interest: The authors declare that the research was conducted in the absence of any commercial or financial relationships that could be construed as a potential conflict of interest.

Publisher's Note: All claims expressed in this article are solely those of the authors and do not necessarily represent those of their affiliated organizations, or those of the publisher, the editors and the reviewers. Any product that may be evaluated in this article, or claim that may be made by its manufacturer, is not guaranteed or endorsed by the publisher.

Copyright (c) 2022 Hassannataj Joloudari, Azizi, Nematollahi, Alizadehsani, Hassannatajjeloudari, Nodehi and Mosavi. This is an open-access article distributed under the terms of the Creative Commons Attribution License (CC BY). The use, distribution or reproduction in other forums is permitted, provided the original author(s) and the copyright owner(s) are credited and that the original publication in this journal is cited, in accordance with accepted academic practice. No use, distribution or reproduction is permitted which does not comply with these terms. 